\title{
A national survey of oral and maxillofacial surgeons' attitudes towards the treatment and dental rehabilitation of oral cancer patients
}

- There has been an increase in the number of oral and maxillofacial surgeons using implants for rehabilitation.

- There has been an increase in the number microvascular tissue grafts used to reconstruct maxillectomy defects over the last 15 years.

- The involvement of a consultant in restorative dentistry in the multidisciplinary cancer team is still limited at $30 \%$

\author{
A. Alani, ${ }^{1}$ J. Owens, ${ }^{2}$ K. Dewan ${ }^{3}$ and A. Summerwill ${ }^{4}$
}

\begin{abstract}
Aims To investigate the attitudes of maxillofacial surgeons in the treatment and dental rehabilitation of oral cancer patients in the UK. Material and methods The survey was conducted by postal questionnaires with 17 close-ended questions. A total of 229 questionnaires were sent to members of the British Association of Oral and Maxillofacial Surgeons over a one week period. A follow-up was sent if a reply was not received within 12 weeks. These results were compared to a similar study that was carried out approximately 15 years ago. Results The response rate was 65.5\% (150/229). Overall $62 \%$ of respondents (92/150) carried out maxillary resections, which represents a decline of $23 \%$ on the previous study. There has been an increase in surgeons reconstructing the maxillary defect from 38\% in the 1995 study to 91\% in the present study. Ninety-eight percent of respondents had their patients seen in a multidisciplinary team (MDT) clinic, but in only 30\% of the cases was a restorative dentist present on these clinics. There has been an improvement in the accessibility of a restorative dentist for this patient cohort, from $65 \%$ to $90 \%$. The use of implants for dental rehabilitation post-cancer surgery has increased from 43\% to 93\%. Conclusion This study highlights the changes in the dental and oral rehabilitation of patients undergoing resective surgery for oral cancer and especially those undergoing a maxillectomy procedure. It illustrates the increased use of implants for post-surgery rehabilitation and shows the different trends in which these implants are placed. An important aspect of this study is the input of the dental team. Current national guidelines state that a consultant restorative dentist needs to be a member of the MDT; this survey shows that this was the case in only $30 \%$ of responses.
\end{abstract}

\section{INTRODUCTION}

The resection of malignant tumours from the palate and maxillary sinus can result in acquired palatal defects. The extent of the defect is dependent on the size, location and behaviour of the tumour. ${ }^{1}$ Post-resection management of the defect can be either primary surgical closure or prosthetic rehabilitation with an obturator. The decision in choosing one of these two treatment modalities is multi-

${ }^{1 *}$ Specialist Registrar in Restorative Dentistry, Department of Restorative Dentistry, Newcastle Dental Hospital, Richardson Road, Newcastle upon Tyne, NE2 $4 \mathrm{AZ} ;{ }^{2}$ Consultant in Restorative Dentistry, Maxillofacial Unit, Morriston Hospital, Heol Maes Eglwys, Morriston, Swansea, SA6 6NL: ${ }^{3}$ Specialist Registrar in Restorative Dentistry, ${ }^{4}$ Consultant in Restorative Dentistry, Department of Restorative Dentistry, Birmingham Dental Hospital, St. Chad's Queensway, Birmingham, B4 6NN ${ }^{*}$ Correspondence to: Mr Aws Alani

Email:aws.alani@nuth.nhs.uk

\section{Online article number E2}

Refereed Paper - accepted 24 August 2009

DOI: 10.1038/sj.bdj.2009.1134

${ }^{\circledR}$ British Dental Journal 2009; 207: E21 factorial and requires a multidisciplinary team approach. ${ }^{2-4}$

The relative advantages and disadvantages of one treatment modality over the other have been discussed. ${ }^{5}$ The main advantages of reconstruction include an immediate closure of the defect with minimal post-operative supervision and the avoidance of nasal reflux that may result from a poorly fitting obturator. ${ }^{6}$ Disadvantages of reconstruction include the difficulty in fabricating a retentive prosthesis over the reconstruction and the difficulties in detecting a tumour recurrence in the resected area. ${ }^{6}$ In contrast, the relative advantages of an obturating prosthesis include the ability to examine the resected site by direct vision, the provision of appropriate lip and cheek support and superior prosthesis retention when utilising the defect undercuts. However, obturators may need frequent adjustments or in many cases, the need for a new obturator soon after initial surgery. ${ }^{6}$ On a psychological level the residual defect may be an uncomfortable reminder of the cancer. ${ }^{7}$

There have been numerous studies measuring the quality of life outcomes of these two treatment modalities. One study found that a well functioning obturator significantly contributed to quality of life post-maxillectomy. ${ }^{8}$ This is in contrast to a later study which found that patients with palatomaxillary reconstruction had superior quality of life in comparison to those prosthetically rehabilitated. ${ }^{9}$ A more recent study found that between the two treatments, no statistically significant difference in quality of life was found. ${ }^{10}$ There seems to be a lack of consensus on how best to rehabilitate this patient cohort.

The need to assess and treat head and neck cancer patients in conjunction with a multidisciplinary team (MDT) has long been cited by Ali et al. ${ }^{6}$ This national survey of maxillofacial surgeons revealed 
that only $65 \%$ had access to a restorative dentist. Interestingly, access to a restorative dentist influenced a maxillofacial surgeon's decision on whether to surgically reconstruct or obturate patients undergoing a maxillectomy procedure. ${ }^{6}$ Since this study there have been numerous national clinical guidelines that outline the need for a restorative dentist to be part of multidisciplinary teams in the management of patients with head and neck cancer. ${ }^{11,12}$

Osseointegrated implants are now a routine consideration when restoring complete and partially edentulous patients. ${ }^{13-15}$ Their use in prosthetically rehabilitating maxillectomy patients is well established. ${ }^{16-18}$ National clinical guidelines have identified those patients undergoing major jaw resection as a priority group for the provision of implants both for intra-oral and extraoral prostheses. ${ }^{19}$ Irrespective of whether the placement of implants for dental rehabilitation in these cases is performed by the restorative dentist or the maxillofacial surgeon, the need for thorough case evaluation and detailed treatment planning is paramount. ${ }^{4}$

When the extent of resection is such that the residual alveolus is insufficient to provide adequate bone for dental implant placement, the use of zygomatic implants can be considered. ${ }^{20,21}$ The original technique was first described by Brånemark in 1998, who published a follow-up over 10 years of 164 implants anchored in zygomatic bone with a success rate of $97 \% .{ }^{22}$ Of note, the patient cohort that was examined in this study did not include oncology patients. Zygomatic implants are considered advantageous in providing an alternative to bone augmentation procedures and so potentially these patients may be fitted with a prosthesis sooner. ${ }^{23} \mathrm{~A}$ notable disadvantage is that patients with zygomatic implants may be at a higher risk of infections that could result in chronic sinusitis. When this occurs it may be necessary to surgically restore ventilation to the sinuses. ${ }^{24}$ A recent systematic review could not identify any suitable studies comparing zygomatic implants and conventional dental implants in augmented bone for severely resorbed maxillae. ${ }^{25}$

In oncology patients who require dental implants, hyperbaric oxygen (HBO) has been advocated by some authors as a prophylactic measure to prevent osteoradionecrosis and improve osseointegration of dental implants. ${ }^{26}$ HBO treatment involves the delivery of $100 \%$ oxygen at high pressure. ${ }^{27}$ It is generally felt that there is a need for better quality of evidence to truly evaluate the benefit of HBO..$^{28,29}$ One randomised trial compared HBO and penicillin in the prevention of osteoradionecrosis (ORN) after dental extractions. The results showed that HBO was more beneficial, with the incidence of ORN in the HBO group markedly less (5.4\%) in comparison to the antibiotic group (29.9\%)..$^{30}$ However, more recent studies have questioned HBO's benefit. In a randomised double-blind trial of patients with ORN treated with either HBO or a placebo, no benefit was seen in the group undergoing hyperbaric oxygenation. ${ }^{31}$ Further to this, oral implant rehabilitation of irradiated patients has been shown to be successful without adjunctive hyperbaric oxygen. ${ }^{32}$

\section{METHODS}

In light of the different options in the treatment of these patients, a questionnaire survey was designed to assess the current practices of consultants in oral and maxillofacial surgery in the UK for patients requiring a maxillectomy procedure (Appendix 1). The main aim was to evaluate the changes in attitudes to the treatment of these patients since this was last charted in a previous study. ${ }^{6}$ In the time that has elapsed since this study there have been numerous clinical guidelines published to outline optimum strategies in the treatment of this patient cohort. ${ }^{11,12,33}$ By repeating the survey, the effect of these publications and guidelines can be estimated.

Names were taken from the list of Fellows of the British Association of Oral and Maxillofacial Surgeons and the questionnaires circulated over a one-week period. A follow-up was sent if no reply was received within 12 weeks.

\section{RESULTS}

A total of 229 questionnaires were sent and 150 were returned, a response rate of $65.5 \%$. Of the 150 questionnaires returned, $61 \%$ (92) of respondents carried out maxillary resections (Table 1 ). This represents a drop of approximately 20\% from the previous study ${ }^{6}$ in which over $80 \%$ of respondents performed maxillectomies (Fig. 1).
The majority of respondents treated between one and five cases a year (55\%), with approximately one third treating between six and ten (33\%) and the remaining more than ten cases (11\%). In the previous study $77 \%$ treated between one and five cases and 19\% of respondents performed between six and ten cases (Table 1, Fig. 2). ${ }^{6}$

Respondents who consistently reconstructed the defect (24\%) were considerably greater in number than those who did not (8.6\%), with the vast majority performing reconstruction sometimes (67\%). In comparison to the previous study, there has been a marked increase in the number of individuals undertaking surgical reconstruction: a rise from 38\% to 91\% (Table 1, Fig. 3). ${ }^{6}$ Further evidence for this is illustrated by the finding that $65 \%$ of respondents reconstructed the defect in 50\% of cases (Table 1, Fig. 4) while in the previous study, 40\% were reconstructing defects in only $10 \%$ of cases. ${ }^{6}$

The most popular type of flap for reconstruction was based on the deep circumflex iliac artery (39\%). This was followed by radial forearm flap (28\%) and then temporalis $(16 \%)$. In comparison to the previous study there has been a marked change in the category of flaps used for reconstruction. ${ }^{6}$ The results show that a greater number of respondents are now favouring microvascular flaps $(80 \%)$ whereas in the previous study rotational flap reconstruction was more popular (64\%) (Table 1, Figs 5 and 6). ${ }^{6}$

Responses to the question on specialties other than maxillofacial surgery performing maxillectomies showed that $27 \%$ of respondents had colleagues in ENT performing this procedure in their trusts, with none from plastic surgery or general surgery (Table 1, Fig. 7).

Responses also showed that while the vast majority (97\%) of respondents had their patients seen by dedicated multidisciplinary teams, the composition of clinicians on these teams was varied (Table 1 , Fig. 8). The vast majority of teams had consultants from ENT (99\%), radiology (91\%), oncology (97\%) and pathology (84\%). Other members who commonly attended the MDT were head and neck specialist nurses (93\%) speech and language therapists (92\%) and dieticians (84\%). In contrast, consultants from restorative 
Table1 Responses to the study questionnaire

Yes

$61.3 \%(92 / 150)$

Do you carry out maxillary resections?

How many cases do you treat a year?

Do you carry out surgical reconstruction of the maxillary defect?

What percentage of cases are surgically reconstructed?

What type of flap do you use to reconstruct the surgical defect?

Do consultants in specialities other than maxillofacial surgery perform maxillectomies regularly in your Trust?

Are all your oncology patients seen on a

multidisciplinary team clinic?

Do you have access to the services of a restorative dentist to assist with prosthetic rehabilitation?

If yes, what grade of dentist do you have in your team?

Following surgical reconstruction, are the patients dentally rehabilitated?

Do you use dental implants to reconstruct patients dentition?

What design of dental implants do you routinely use for prosthetic reconstruction?

Are the implants placed by the surgeon or the restorative dentist?

Are dental implants placed at the time of primary surgery?

Do you use zygomatic implants for maxillary $p$ rosthetic reconstruction?

Do you have access to hyperbaric oxygen therapy (HBO) for patients with osteoradionecrosis or requiring dental implants?

Do you routinely use HBO for patients who have undergone radiotherapy who are scheduled for dental implants?

\begin{tabular}{|c|c|c|c|c|c|}
\hline \multicolumn{3}{|c|}{$61.3 \%$ (92/150) } & \multicolumn{3}{|c|}{$38.7 \%(58 / 150)$} \\
\hline \multicolumn{2}{|c|}{ Between 1-5 } & \multicolumn{2}{|c|}{ Between 6-10 } & \multicolumn{2}{|c|}{ Over 10} \\
\hline \multicolumn{2}{|c|}{$55.4 \%(51 / 92)$} & \multicolumn{2}{|c|}{$32.6 \%(30 / 92)$} & \multicolumn{2}{|c|}{$11.9 \%(11 / 92)$} \\
\hline \multicolumn{2}{|c|}{ Yes } & \multicolumn{2}{|c|}{ Sometimes } & \multicolumn{2}{|c|}{ Never } \\
\hline \multicolumn{2}{|c|}{$23.9 \%$ (22/92) } & \multicolumn{2}{|c|}{$67.3 \%(62 / 92)$} & \multicolumn{2}{|c|}{$8.6 \%(8 / 92)$} \\
\hline \multicolumn{2}{|c|}{$0-25 \%$} & $5-50 \%$ & \multicolumn{2}{|c|}{$50-75 \%$} & $75-100 \%$ \\
\hline \multicolumn{2}{|c|}{$35.8 \%(33 / 92)$} & $29.3 \%(27 / 92)$ & \multicolumn{2}{|c|}{$18.4 \%(17 / 92)$} & $16.3 \%(15 / 92)$ \\
\hline $\begin{array}{c}\text { Radial forearm } \\
\text { graft }\end{array}$ & Temporalis & $\begin{array}{c}\text { Deep } \\
\text { circumflex } \\
\text { iliac artery }\end{array}$ & Scapula & Fibula & Others \\
\hline $28 \%(26 / 92)$ & $16 \%(15 / 92)$ & $39 \%(36 / 92)$ & $9 \%(8 / 92)$ & $4 \%(4 / 92)$ & $4 \%(3 / 92)$ \\
\hline \multicolumn{2}{|l|}{ No } & ENT & \multicolumn{2}{|c|}{ Plastics } & General surgery \\
\hline \multicolumn{2}{|c|}{$73 \%(71 / 97)$} & $6.8 \%(26 / 97)$ & \multicolumn{2}{|c|}{$0 \%(0 / 97)$} & $0 \%(0 / 97)$ \\
\hline \multicolumn{2}{|c|}{ Yes } & \multicolumn{2}{|c|}{ No } & \multicolumn{2}{|c|}{ Sometimes } \\
\hline \multicolumn{2}{|c|}{$97.8 \%(90 / 92)$} & \multicolumn{2}{|c|}{$2.2 \%(2 / 92)$} & & \\
\hline Yes, & vays & Yes, s & mes & & \\
\hline $73.9 \%$ & 8/92) & 16.3 & 92) & & 92) \\
\hline $\begin{array}{l}\text { Consultant } \\
\text { restorative } \\
\text { dentist }\end{array}$ & $\begin{array}{l}\text { Associate } \\
\text { specialist }\end{array}$ & Staff grade & $\begin{array}{l}\text { Clinical } \\
\text { assistant }\end{array}$ & $\mathrm{SHO}$ & Other \\
\hline $73 \%$ (60/83) & $11 \%(9 / 83)$ & $8 \%(7 / 83)$ & $5 \%(4 / 83)$ & $2 \%(2 / 83)$ & $1 \%(1 / 83)$ \\
\hline
\end{tabular}

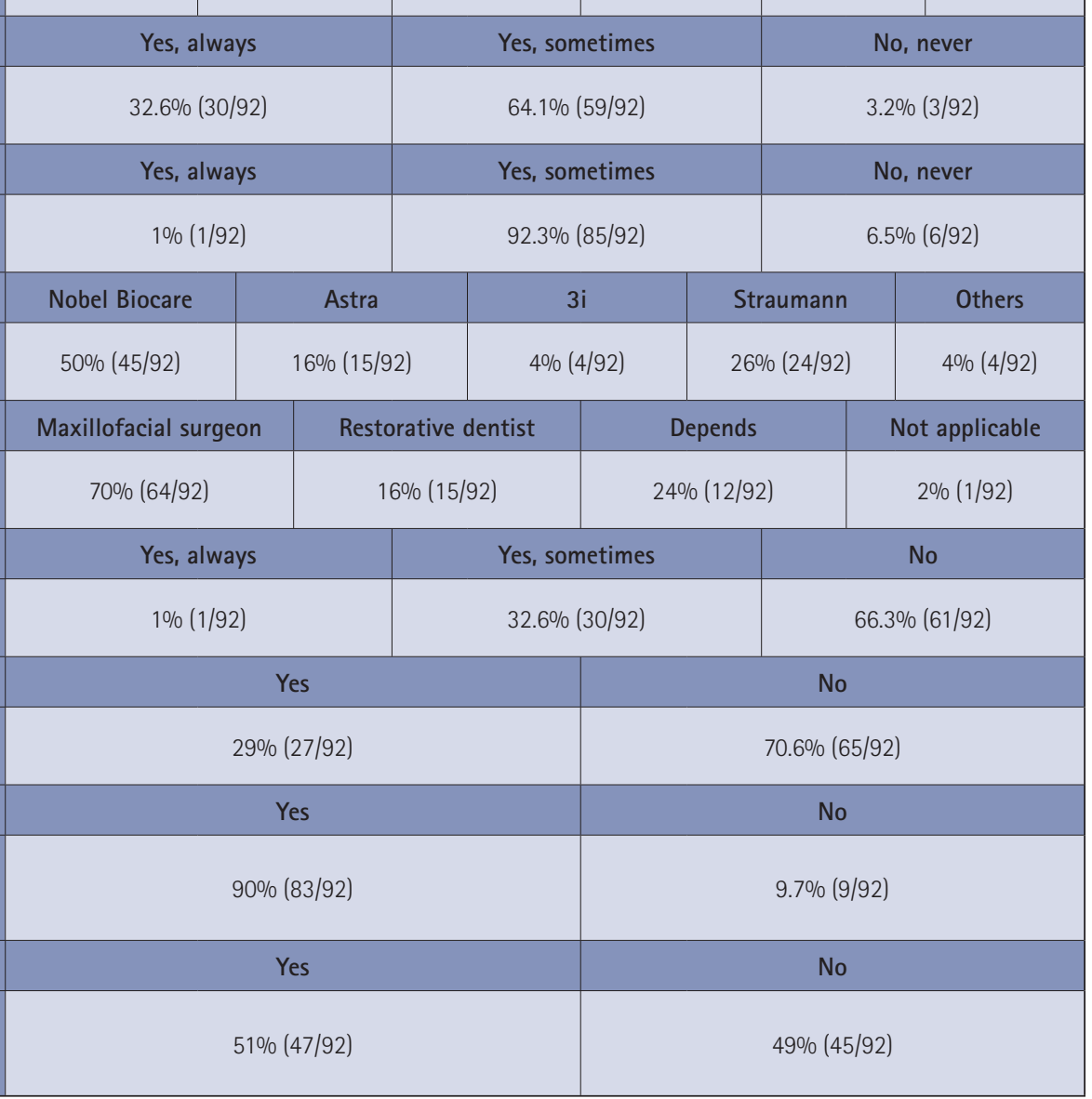


dentistry (32\%), palliative care (41\%) and dental hygienists (23\%) were less common (Fig. 9).

Nearly three quarters of respondents had access to a restorative dentist (74\%) with considerably less having access sometimes (16\%) and never (9\%) (Table 1). In total $90 \%$ had access to a restorative dentist, which has increased from 65\% in the previous study (Fig. 10). ${ }^{6}$ When access to a restorative dentist was available the grade of the clinician varied. The majority were consultants (73\%), with associate specialists (11\%) staff grades (8\%), clinical assistants (5\%) and senior house officers (2\%) comprising the group of other dental professionals inputting into the planning process (Table 1, Fig. 11). Following reconstruction, the responses on dental rehabilitation post-surgery varied. Thirty-three percent indicated that patients always underwent dental rehabilitation and $64 \%$ indicated this sometimes occurred, while the proportion indicating patients were never rehabilitated was small (3\%) (Table 1, Fig. 12).

The use of implants in dental rehabilitation was performed always by $1 \%$ of respondents and sometimes by $92 \%$, with the remaining 6.5\% not using implants for this purpose (Table 1, Fig. 13). This is a marked increase on the previous study where $43 \%$ of respondents were using implants for rehabilitation. The types of implants used included Nobel Biocare (Zurich, Switzerland) (50\%) and Straumann (Basel, Switzerland) (26\%) (Table 1, Fig. 14). The different types of implants used have changed from the previous study. Previously 30\% of respondents were using 'Bonefit' implants (product line of Straumann implants in 1995) and 39\% were using 'Brånemark' implants (Nobel Biocare Implants) (Fig. 15). Astra Tech implants (Mölndal, Sweden) have also increased in their usage from $12 \%$ to $16 \%$ and there has been an emergence of newer implant systems such as Biomet $3 \mathrm{i}$ (Palm Beach Gardens, Florida), with 3\% of respondents using this implant system in this study and no significant recordings in 1995.

The placement of the implants for the purpose of oral rehabilitation was most commonly performed by the maxillofacial surgeon $(70 \%)$ or the restorative dentist (16\%) and in $24 \%$ of responses

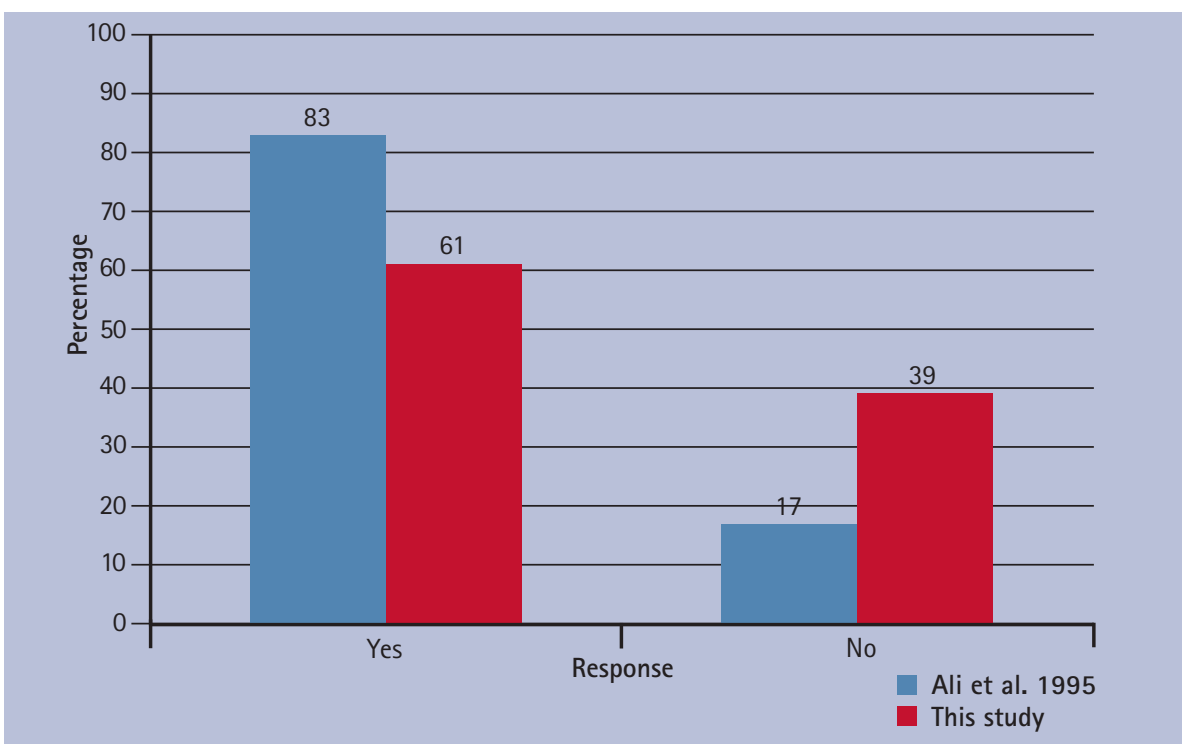

Fig. 1 The percentage of respondents carrying out maxillary resections. Comparison between the present study and a previous study ${ }^{6}$

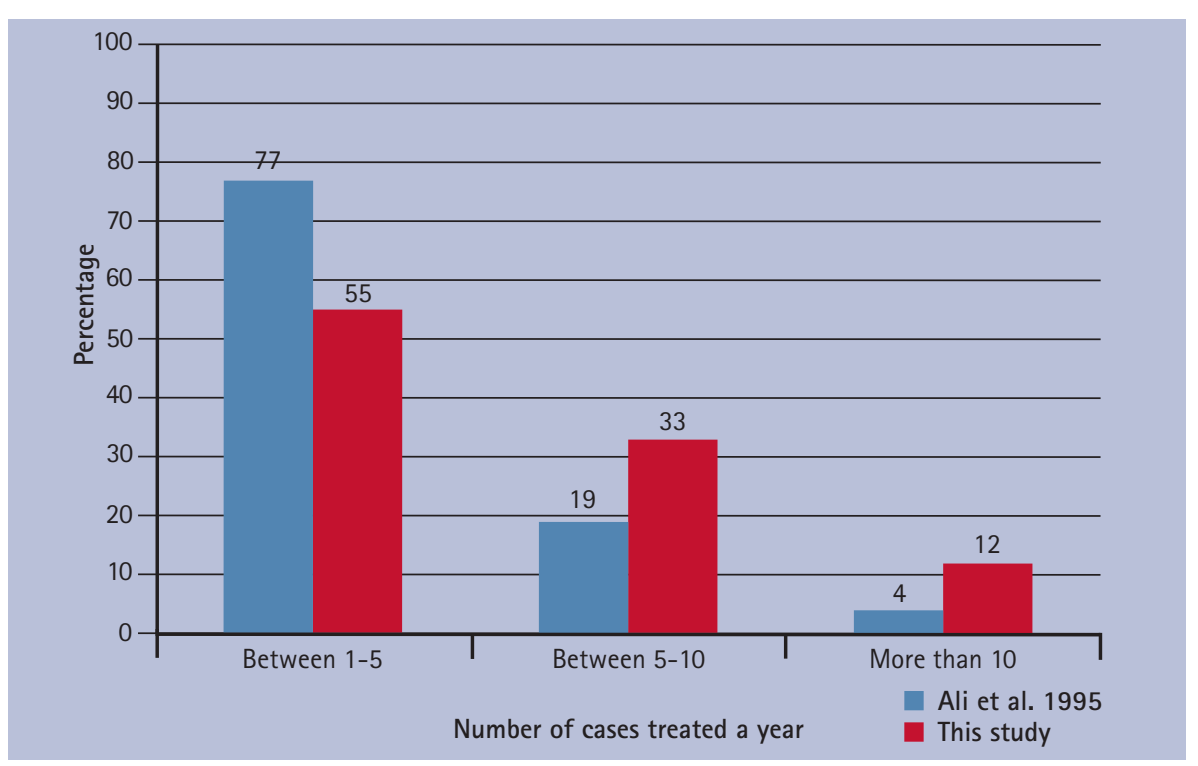

Fig. 2 Number of cases treated a year in the previous study ${ }^{6}$ and the present study

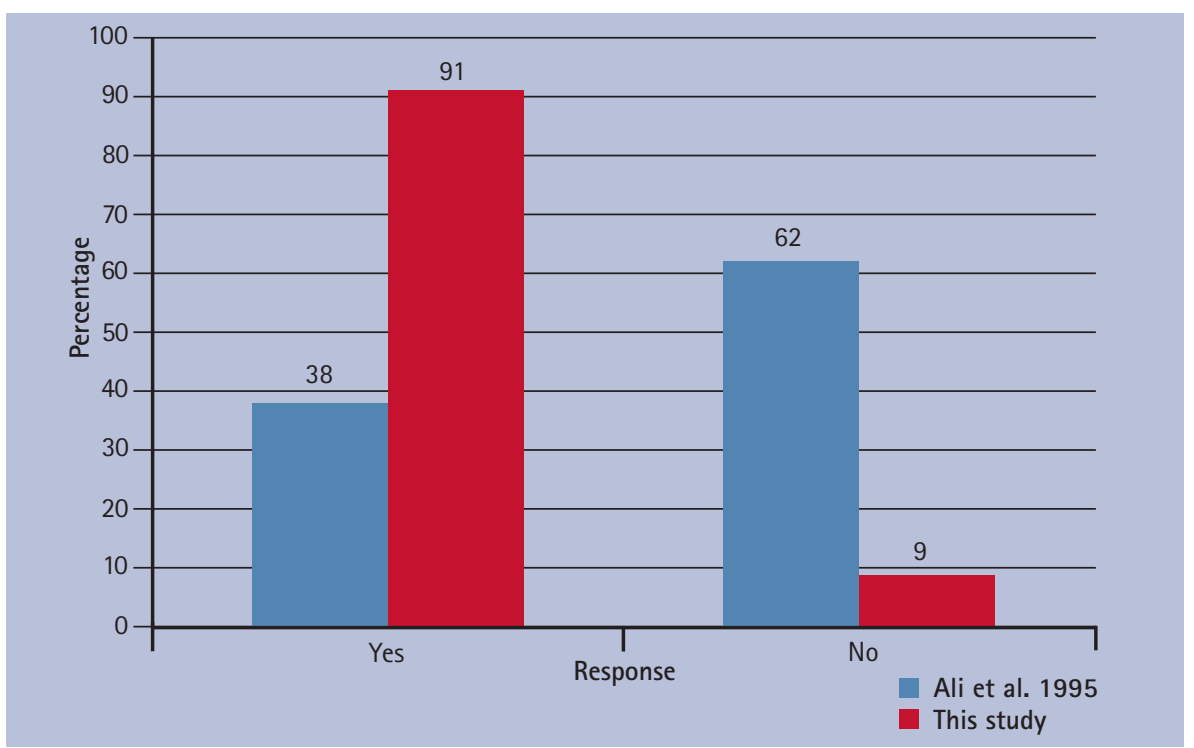

Fig. 3 Respondents carrying out surgical reconstruction of the defect in the previous study ${ }^{6}$ and the present study 


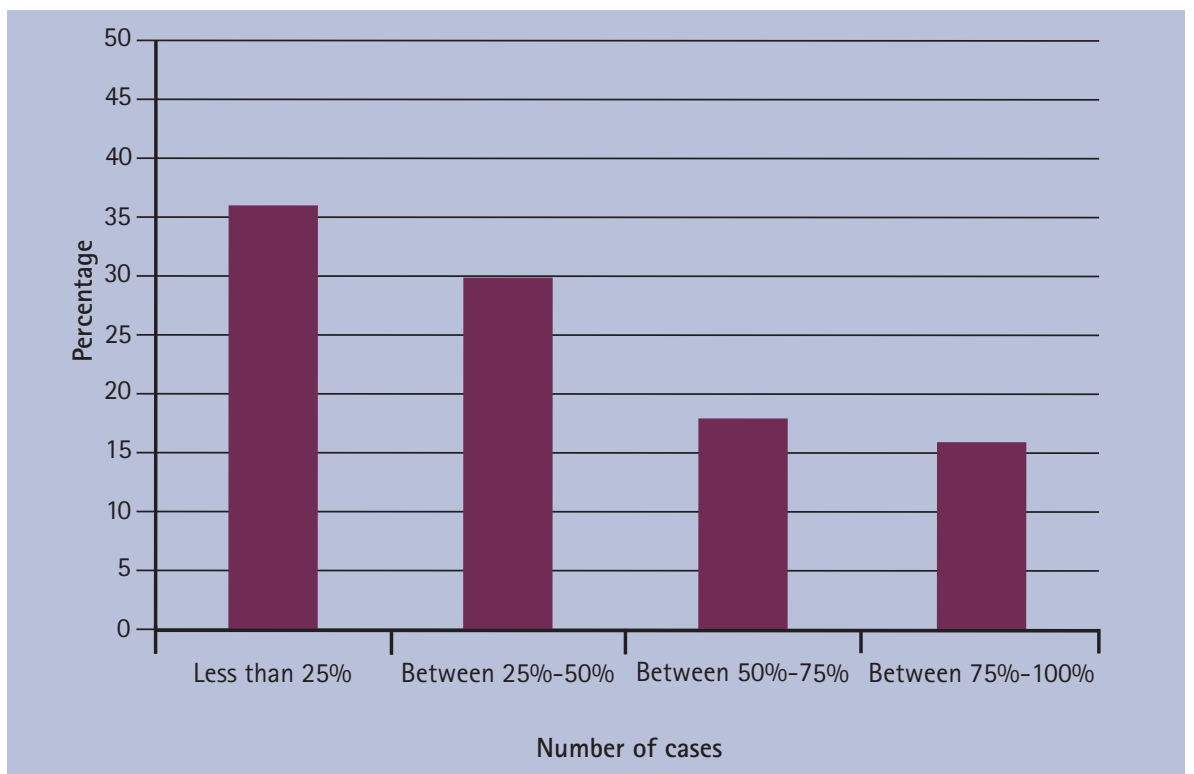

Fig. 4 Column graph of surgical reconstruction

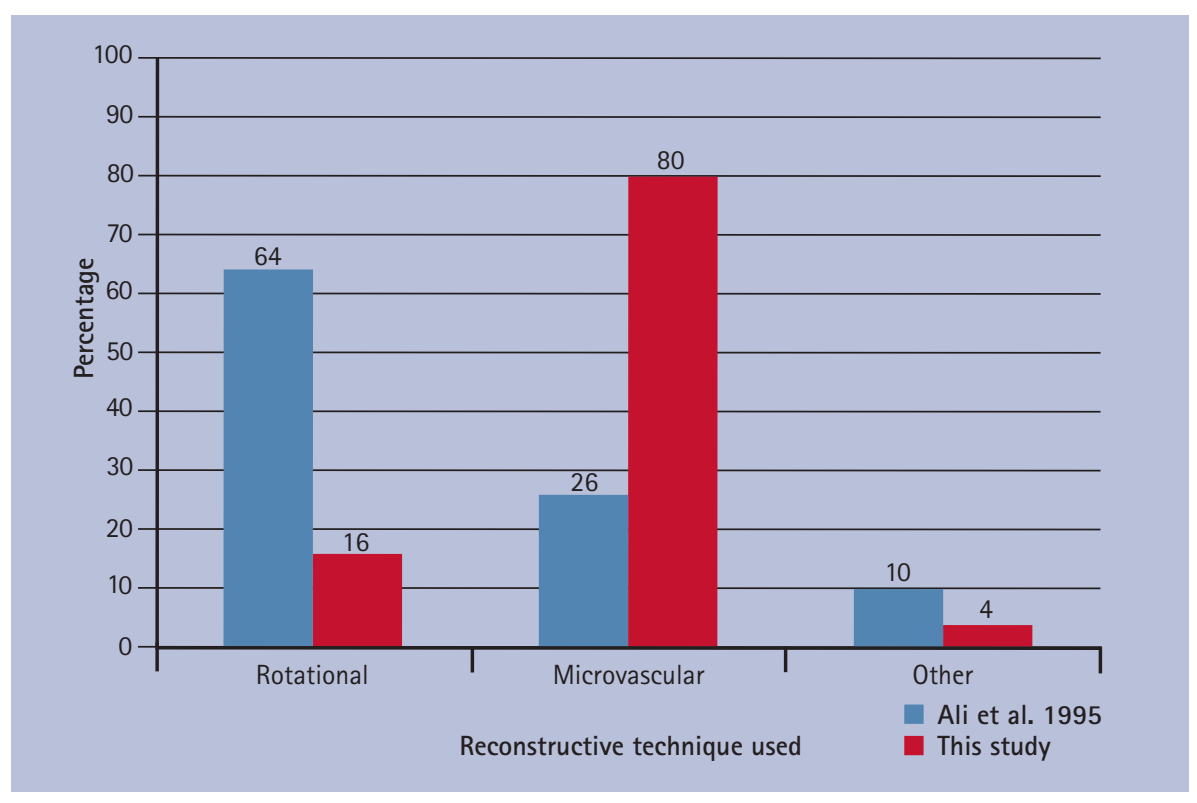

Fig. 6 Column graph comparing the differences in categories of flap designs (rotational, microvascular or other) between the previous study ${ }^{6}$ and the present study

the decision depended on the type of case (Table 1, Fig. 16). Implant placement at time of ablative surgery occurred sometimes in 33\% of responses and not at all in $66 \%$, with $1 \%$ performing this always (Table 1, Fig. 17). Twenty-nine percent of respondents placed zygomatic implants for maxillary prosthetic reconstruction, while the majority (71\%) did not (Table 1 , Fig. 18).

Access to hyperbaric oxygen was available to $90 \%$ of respondents (Table 1, Fig. 19). Fifty-one percent routinely used this for patients undergoing radiotherapy and requiring implants (Table 1 , Fig. 20).

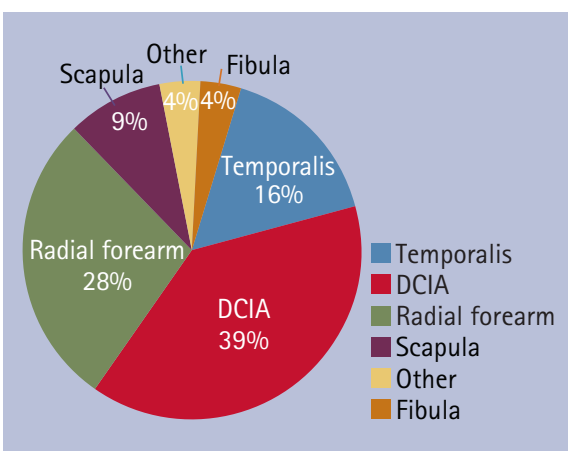

Fig. 5 Pie chart representing the choice of flaps chosen by the respondents for surgical reconstruction

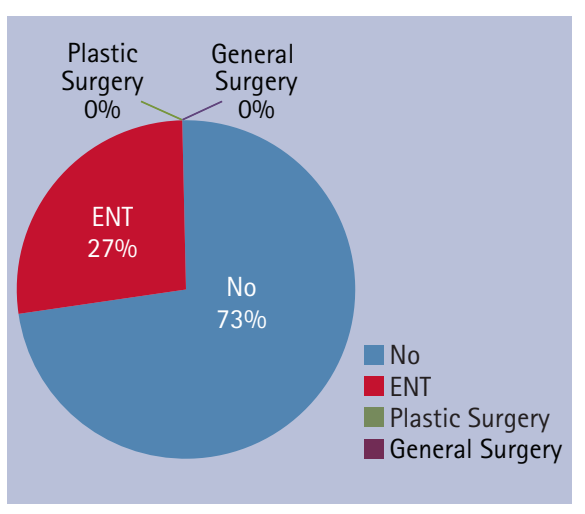

Fig. 7 A pie chart representing the proportions of other specialties performing maxillectomies

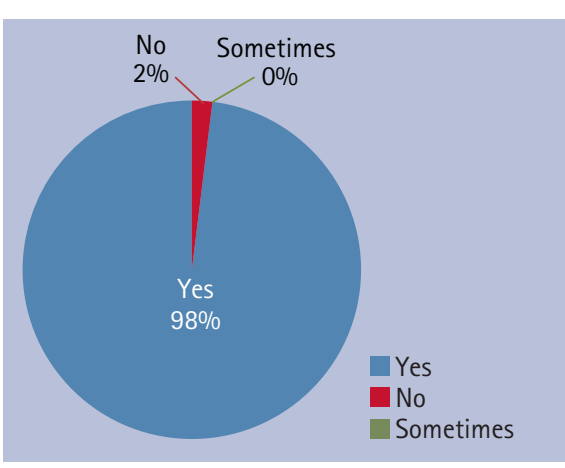

Fig. 8 Pie chart representing access to multidisciplinary clinics

a year, from $3 \%$ to $11 \% .^{6}$ These results may signify the development of niche skills by individuals within the specialty, with the development of surgeons whose work is primarily dedicated to a cancer multidisciplinary team.

This concept is supported by $98 \%$ of respondents working in such a team (Fig. 8). This high percentage is probably a reflection of the Calman and Hine report ${ }^{33}$ and the more recent SIGN and NICE guidelines identifying the need for MDTs in the management of head and neck cancer. ${ }^{11,12}$ More specifically these guidelines outline the need for a restorative dentistry consultant to be part of the MDT. Our results 
show that there has been an increase in respondents' access to restorative dentists from $65 \%{ }^{6}$ rising to $90 \%$ in the present study, although the grade of clinician varied from senior house officers to consultant (Fig. 11). In spite of this improvement, the perceived ideal of a fully integrated consultant in restorative dentistry present on the multidisciplinary team was recorded in only $30 \%$ of responses.

In the present study $24 \%$ of respondents always carried out surgical reconstruction of the defect, with the majority carrying out reconstruction 'sometimes' (67\%). This is in contrast to the previous study where 38\% of cases were reconstructed. These observations could signify a paradigm shift in the management of patients requiring maxillary resection. A recent retrospective study of maxillary reconstructions between 1992 and 2001 showed that $84 \%$ of cases were reconstructed surgically with $16 \%$ being rehabilitated with an obturator prosthesis. ${ }^{34}$ The increased popularity of microsurgical techniques and the increased use of implants for prosthetic rehabilitation in post-surgical reconstruction could coincide with a decrease in the need for traditional prosthetic obturator provision. ${ }^{34,35}$

of the flaps used to reconstruct maxillary defects, the temporalis flap was the most common (53\%) in the 1995 study, while in the present study this has dropped to $19 \%$. This has coincided with a marked increase in the use of microvascular techniques (from 26\% to 80\%) and a decrease in rotational flaps (from $64 \%$ to $16 \%$ ) in post-resective surgical reconstruction (Fig. 6). This large variation in techniques could reflect the different extent of maxillary resections, with certain techniques being only suitable for smaller defects.

The use of implants in the reconstructive phase of treatment was undertaken by over $90 \%$ of the respondents, in comparison to the previous study where there were $43 \%$. The increase in implant usage for rehabilitation is probably due to the increased availability and training in implant provision for maxillofacial surgery and restorative dentistry trainees and the increased involvement of restorative dentists in the MDT. Other factors include guidelines identifying patients with major jaw resections as priority groups for implant rehabilitation..$^{19,36,37}$ Previous studies have

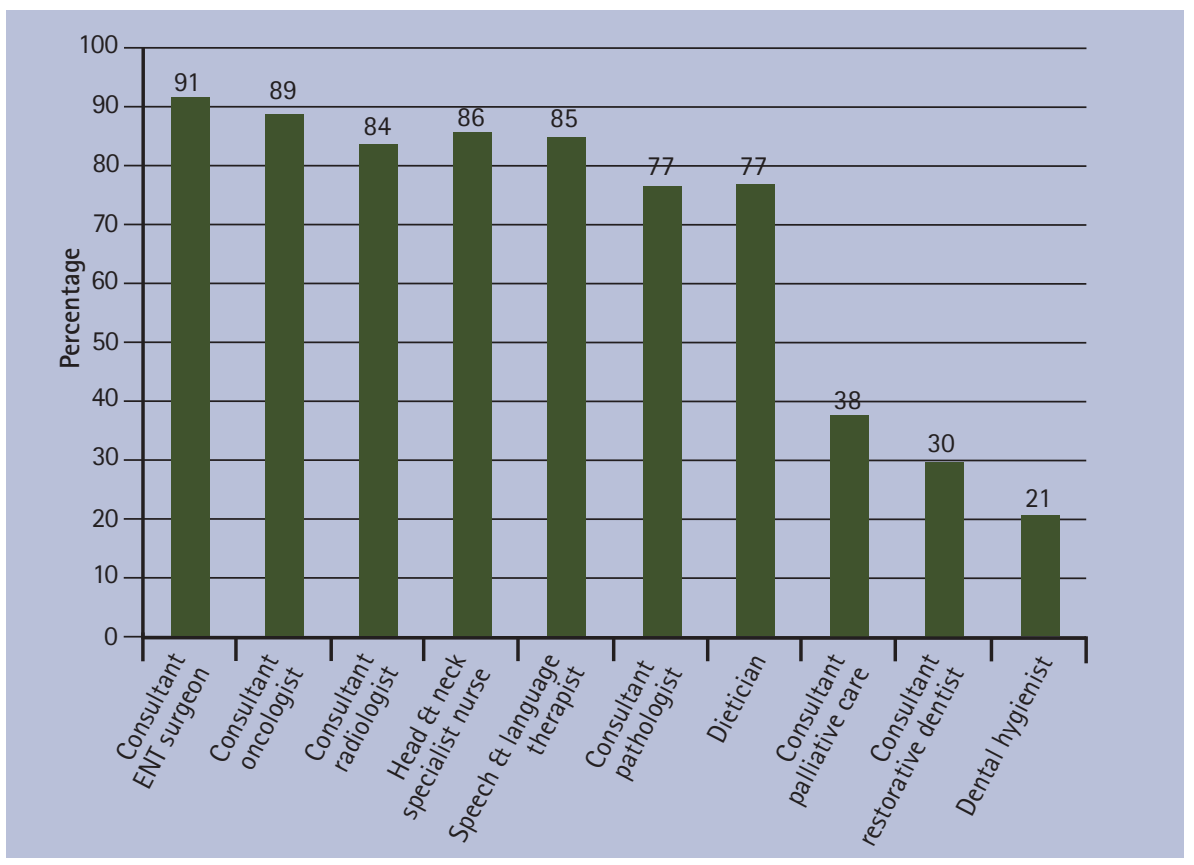

Fig. 9 Graph representing the professional composition of multidisciplinary teams

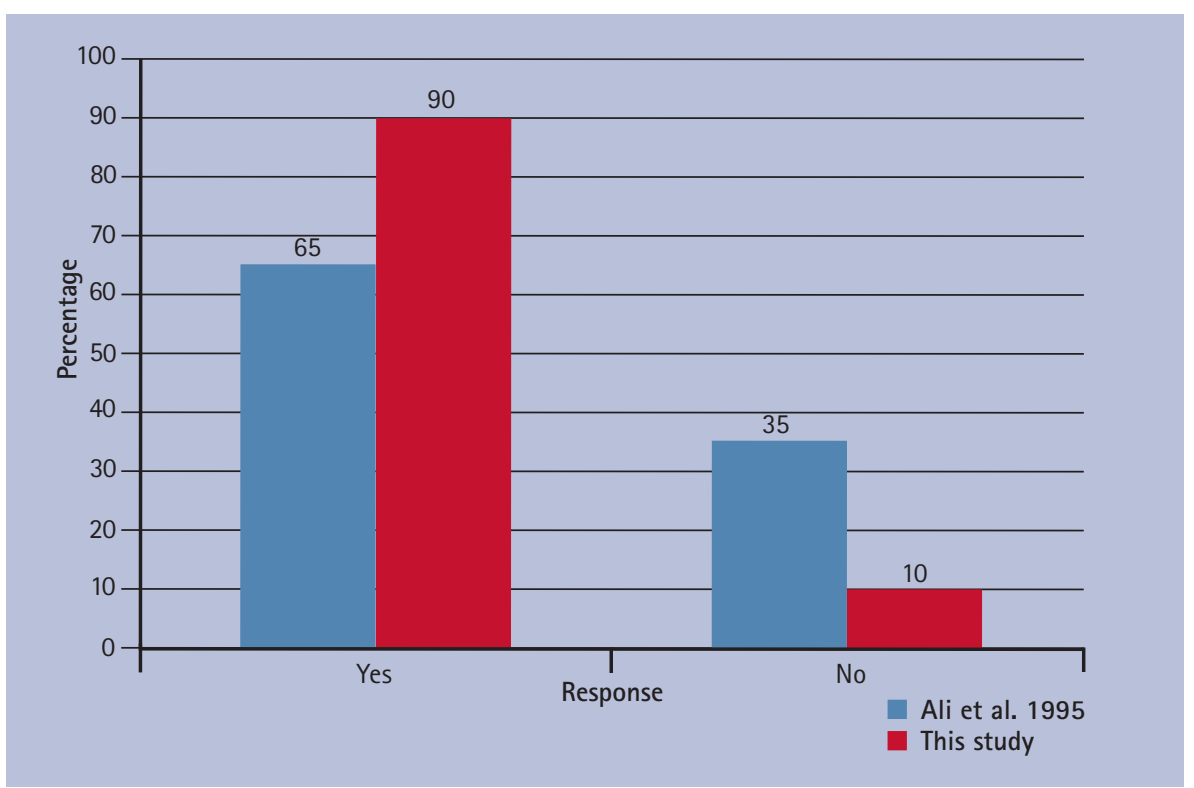

Fig. 10 Graph representing access to a restorative dentist for the purpose of oral rehabilitation

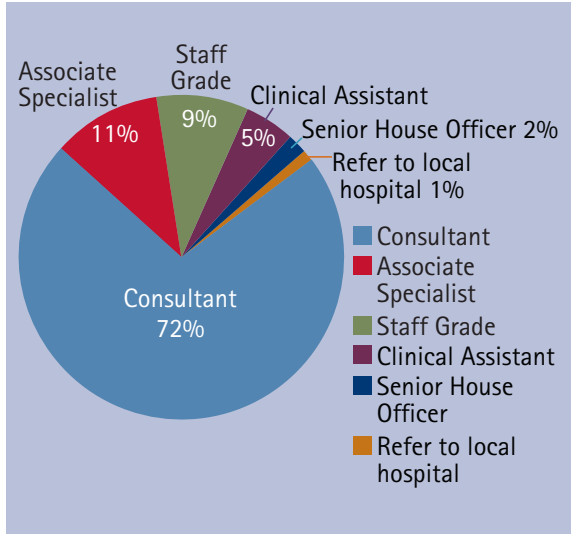

Fig. 11 Pie chart representing proportions of restorative dentist grades

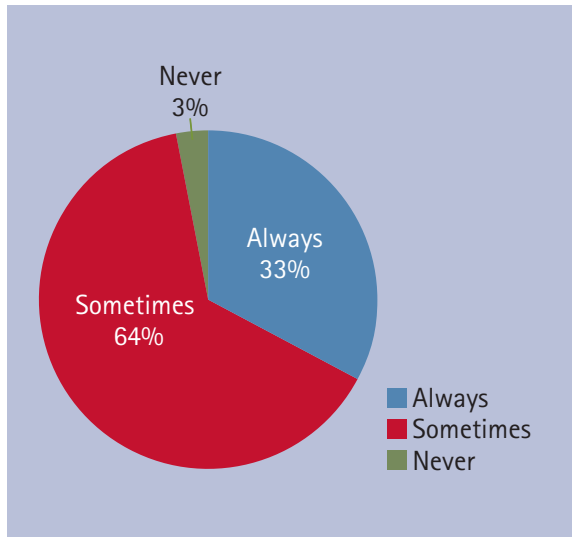

Fig. 12 Pie chart representing responses on the dental rehabilitation of patients 


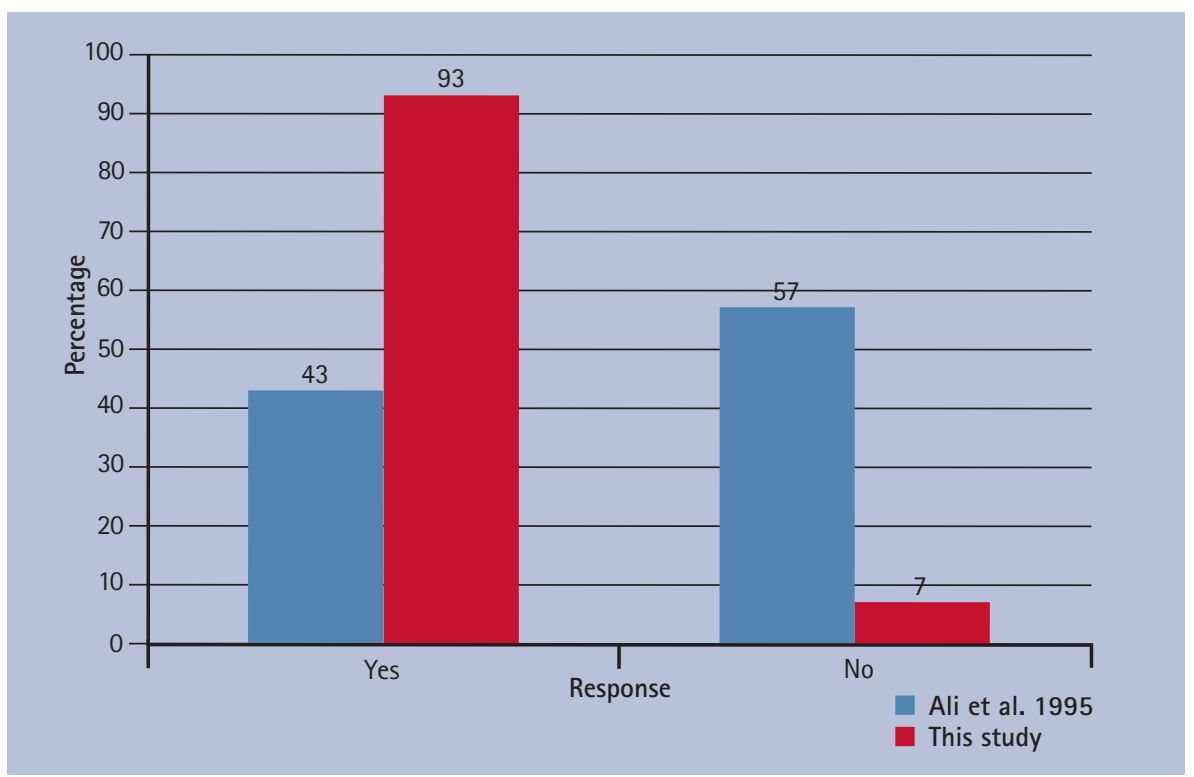

Fig. 13 Block graph representing the use of implants in oral rehabilitation

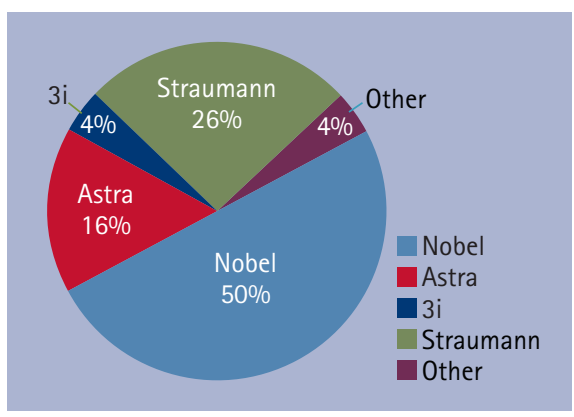

Fig. 14 Pie chart representing the types of implants used by respondents

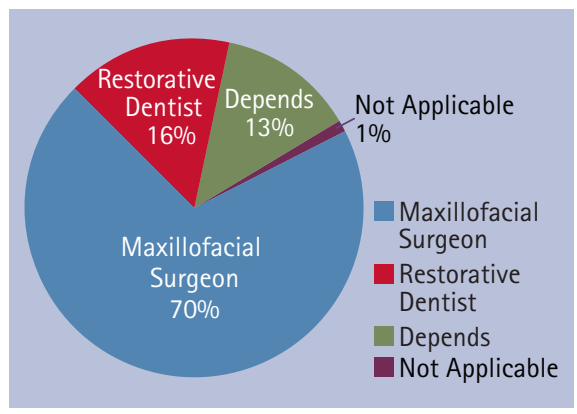

Fig. 16 Clinicians placing the implants in oral rehabilitation

shown that there is a marked variation in the number of patients treated with dental implants within National Health Service hospitals but in most cases this follows the Royal College of Surgeons guidelines of $1997 . .^{38,39}$

The increase in the spread of different types of implants in the present study reflects the emergence of numerous companies developing and producing new implant systems since 1995. The most popular system used was Nobel Biocare (49\%) followed by Straumann (26\%). The most

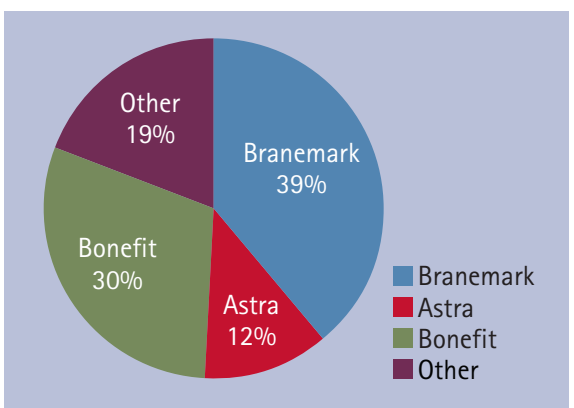

Fig. 15 Design of implants from the previous study ${ }^{6}$

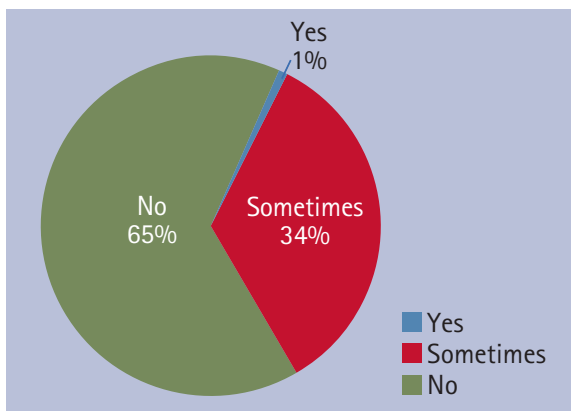

Fig. 17 Placement of implants at the time of surgery

popular system in the 1995 study was the Brånemark implant (39\%) (now branded Nobel Biocare). ${ }^{6}$ The use of different types of implants by operators may be irrelevant as a recent systematic review found there is not enough evidence from randomised controlled trials to demonstrate the superiority of any particular type of implant design or implant system over the others. ${ }^{40}$ As technology and research into implant coatings progress, the likelihood of popular implant designs at present becoming defunct in the future needs to be appreciated. This is

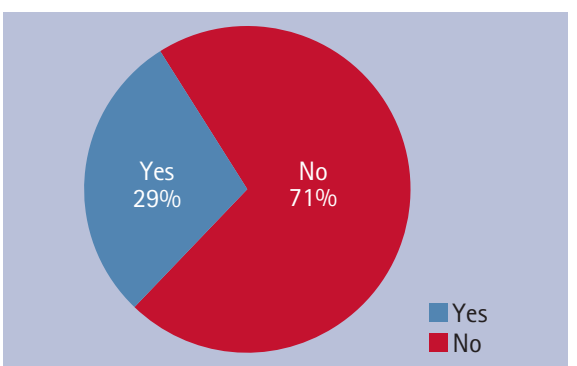

Fig. 18 Pie chart illustrating the use of zygomatic implants for dental rehabilitation

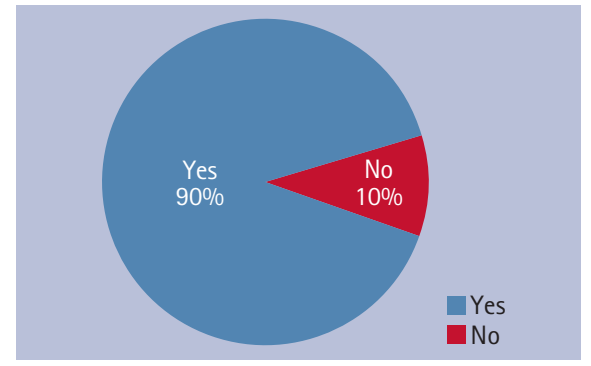

Fig. 19 Access to hyperbaric oxygen for dental rehabilitation or osteoradionecrosis

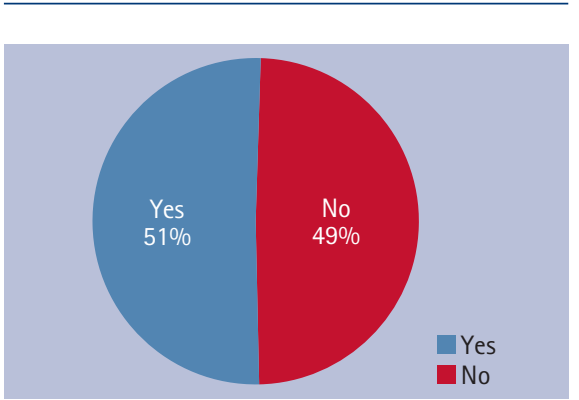

Fig. 20 Routine use of $\mathrm{HBO}$ for patients who have undergone radiotherapy who are scheduled for dental implants

illustrated by $30 \%$ of respondents in the original study using 'Bonefit' implants, which are plasma sprayed. Since then there has been research into implant coatings especially at the "nanostructural' level.,41 As survival rates for oral cancer continually improve and with the constant turnover and emergence of new implant systems, the provision of implant recognition systems for discontinued as well as new implant systems in the long term management of this patient cohort is important. ${ }^{42,43}$

The placement of the implants yielded interesting results. Sixty-four percent of maxillofacial surgeons placed the implants for the purpose of oral rehabilitation, in comparison to $15 \%$ placed by the restorative dentist. The clinician placing the implants depended on the situation in some cases (22\%). As the purpose of implants in these cases, where normal oral anatomy has changed sometimes radically, is for the 
restoration of oral function and aesthetics, the need for multidisciplinary team interaction in the planning of the position of fixtures is vital.

The apparent discrepancy between patients either being rehabilitated sometimes or never (67\%) in comparison to the percentage of surgeons using implants for dental rehabilitation (93\%) could be indicative of a gap between what is technically possible but, due to other constraints, unable to be routinely offered. The placement of implants in this cohort ultimately requires the knowledge and skill to provide associated restorations to a specialist level. As our results show that the presence of a restorative dentist on the MDT is still at a minimum (30\%), other barriers such as funding and training may also be present. This area requires further investigation.

The placement of implants at the time of surgery was not routinely considered in $66 \%$ of responses. The relative advantages of immediate implant placement include preventing the need for a second episode of surgery, the possible need for adjunctive HBO therapy and preventing placement of implants in irradiated tissue. ${ }^{44}$ The relative disadvantages of placement at the ablative stage include the difficulty in assessing the prognosis of the patient and as such the possible need for more surgery if initial treatment is unsuccessful. A study examining implant survival of 435 implants in 93 patients post-resective surgery showed that the mean survival rate (69\%) at ten years was lower than in healthy individuals. This was attributed to the higher mortality rate of the cohort as opposed to failed osseointegration. Of note is the fact that the cumulative survival rate for fixtures in the maxilla was $72 \%$ in comparison to $92 \%$ in the mandible, and all implants were placed at least six months post-radiotherapy. ${ }^{45}$ It seems that the best options in these situations will invariably require a team approach to the time, type and also the position of implant placement.

Over 70\% of respondents used zygomatic implants, and their value in preventing the need for bone grafting in rehabilitating naturally atrophic maxillas, patients with severe bone loss secondary to trauma and extensive bilateral maxillectomy procedures, cannot be overestimated. ${ }^{23}$ Utilising zygomatic bone for osseointegration in these cases prevents the need for a wide range of surgical interventions which necessitate morbidity of an extraoral donor site, patients remaining without a prosthesis during the graft consolidation phase and healing time. Schmidt and coworkers conducted a retrospective study of 28 zygomatic implants in patients with total maxillectomies. Six of the zygomatic implants placed failed (78\% success rate) ${ }^{46}$ The results of another study yielded similar results in a retrospective study of 28 zygomatic implants placed in patients who previously underwent maxillary resection, with a success rate of $71 \% .{ }^{47}$ On the evidence of these studies it would seem that the placement of zygomatic implants in post-maxillectomy cases has a lower success rate than in otherwise healthy individuals. This difference in success rate was attributed to the use of radiotherapy in this patient cohort, which may have also included smokers.

Over $90 \%$ of respondents had access to hyperbaric oxygen for patients with osteoradionecrosis or those requiring implants. Fifty percent of respondents did not routinely use HBO for patients who underwent radiotherapy and were having implants placed. This may reflect the current uncertainty on the benefit of HBO. A Cochrane review ${ }^{48}$ found one suitable randomised controlled trial that compared one group of HBO treated patients with another who received no HBO for implant treatment in radiated patients. In this study patients treated with $\mathrm{HBO}$ performed worse in every aspect when compared to patients not subjected to HBO therapy. ${ }^{49}$ Eight implants failed in five patients subjected to HBO therapy versus three implants in two patients in the control group. Two postoperative complications (one osteoradionecrosis and the other soft tissue complications) developed in two patients subjected to HBO therapy. ${ }^{49}$ The conclusion of the systematic review, with consideration to the limited amount of research available, is that hyperbaric oxygen (HBO) therapy in irradiated patients requiring dental implants may not offer any appreciable clinical benefits. ${ }^{48}$ As it is difficult to obtain the best quality of evidence in such a patient cohort, research into the relative benefits of HBO will need further investigation as at present there seem to be differing opinions on its benefit. A recent survey of maxillofacial surgeons revealed that most consider HBO to be part of the management of osteoradionecrosis, but their knowledge about delivery was weak and the protocols used varied. ${ }^{29}$

\section{CONCLUSION}

This survey gives an insight into the changes that have occurred in the treatment of head and neck cancer patients, in particular those undergoing maxillectomy, over the last ten years. The period has coincided with the production of a number of policy documents and guidelines designed to improve the quality of these services.

It highlights a growth in multidisciplinary team working but also shows variation in the utilisation of care team members across trusts and across specialties. In particular there appears to be a significant discrepancy in utilisation of restorative team members and how and at what level they input into the care of head and neck oncology patients.

Over this period there has been a significant growth in the use of more complex microvascular free-flaps for the reconstruction of maxillectomy patients. There appears to be no consensus, however, as to the 'ideal' reconstruction technique. While conventional prosthetic rehabilitation still has an important role to play for oncology patients, there has been a significant increase in the use of dental and zygomatic implants in their rehabilitation.

An area of major contention is the management of these patients and the use of hyperbaric oxygen in aiding osseointegration of dental implants. This highlights the lack of good scientific evidence for or against the treatment modality and therefore the need for more randomised controlled studies.

While overall the results of the study are encouraging, they show the need for further resources to attain the ideal standards across NHS trusts and specialties.

The authors would like to thank the members of the British Association of Oral and Maxillofacial Surgeons for completing and returning the questionnaires.

1. Triana R J Jr, Uglesic V, Virag M et al. Microvascular free flap reconstructive options in patients with partial and total maxillectomy defects. Arch Facial Plast Surg 2000; 2: 91-101.

2. Friedman E, Friedman C. Tumours of the head and neck. A 4-year study of a multidisciplinary approach. Int J Oral Surg 1978; 7: 291-295.

3. King G E, Lemon J C, Martin J W. Multidisciplinary teamwork in the treatment and rehabilitation of 
the head and neck cancer patient. Tex Dent J 1992; 109: 9-12.

4. Okay D J, Genden E, Buchbinder D, Urken M. Prosthodontic guidelines for surgical reconstruction of the maxilla: a classification system of defects. J Prosthet Dent 2001; 86: 352-363.

5. Pigno M A. Conventional prosthetic rehabilitation after free flap reconstruction of a maxillectomy defect: a clinical report. J Prosthet Dent 2001: 86: $578-581$.

6. Ali A, Fardy M J, Patton D W. Maxillectomy - to reconstruct or obturate? Results of a UK survey of oral and maxillofacial surgeons. Br J Oral Maxillofac Surg 1995; 33: 207-210

7. Patton D W, Ali A, Davies R, Fardy M J. Oral rehabilitation and quality of life following the treatment of oral cancer. Dent Update 1994; 21: 231-234.

8. Kornblith A B, Zlotolow I M, Gooen J et al. Quality of life of maxillectomy patients using an obturator prosthesis. Head Neck 1996; 18: 323-334.

9. Genden E M, Okay D, Stepp M T et al. Comparison of functional and quality-of-life outcomes in patients with and without palatomaxillary reconstruction: a preliminary report. Arch Otolaryngol Head Neck Surg 2003; 129: 775-780.

10. Rogers S N, Lowe D, McNally D, Brown J S, Vaughan E D. Health-related quality of life after maxillectomy: a comparison between prosthetic obturation and free flap. J Oral Maxillofac Surg 2003; 61: 174-181.

11. National Institute for Clinical Excellence. Guidance on cancer services: Improving outcomes in head and neck cancers: the manual. London: NICE, 2004

12. Scottish Intercollegiate Guidelines Network. Diagnosis and management of head and neck cancer: a national clinical guideline. Edinburgh: SIGN, 2006

13. Brånemark $\mathrm{PI}$, Hansson B $\mathrm{O}$, Adell $\mathrm{R}$ et al. Osseointegrated implants in the treatment of the edentulous jaw. Experience from a 10-year period. Scand J Plast Reconstr Surg Supp/ 1977; 16: 1-132.

14. Smith D E. Review of endosseous implants for partially edentulous patients. Int J Prosthodont 1990; 3: $12-19$

15. Jemt $T$, Lekholm U. Oral implant treatment in posterior partially edentulous jaws: a 5-year follow-up report. Int J Oral Maxillofac Implants 1993; 8: 635-640.

16. Devlin $H$, Barker G R. Prosthetic rehabilitation of the edentulous patient requiring a partial maxillectomy. J Prosthet Dent 1992; 67: 223-227.

17. Kabcenell J, Silken D, Kraut R. Restoration of a total maxillectomy patient using endosseous implants. Int J Prosthodont 1992; 5: 179-183.

18. Lorant J A, Roumanas E, Nishimura R, Beumer J 3rd, Wagman LD. Restoration of oral function after maxillectomy with osseous integrated implant retained maxillary obturators. Am J Surg 1994; 168: 412-414.

19. Faculty of Dental Surgery of the Royal College of Surgeons of England. National Clinical Guidelines 1997. Restorative Dentistry section: Guidelines for selecting appropriate patients to receive treatment with dental implants: priorities for the NHS. London: Royal College of Surgeons of England, 1997.

20. Weischer T, Mohr C. Ten-year experience in oral implant rehabilitation of cancer patients: treatment concept and proposed criteria for success. Int J Oral Maxillofac Implants 1999; 14: 521-528.

21. Tamura $H$, Sasaki $K$, Watahiki R. Primary insertion of implants in the zygomatic bone following subtotal maxillectomy. Bull Tokyo Dent Coll 2000; 41: 21-24.

22. Brånemark P-I. Surgery and fixture installation. In Zygomaticus fixture clinical procedures. 1st ed. pp 1. Goteborg, Sweden: Nobel Biocare AB, 1998.

23. Urgell J P, Gutiérrez V R, Escoda C G. Rehabilitation of atrophic maxilla: a review of 101 zygomatic implants. Med Oral Patol Oral Cir Bucal 2008;13: E363-E370.

24. Petruson B. Sinuscopy in patients with titanium implants in the nose and sinuses. Scand J Plast Reconstr Surg Hand Surg 2004; 38: 86-93.

25. Esposito $M$, Worthington $H$ V, Coulthard $P$. Interventions for replacing missing teeth: dental implants in zygomatic bone for the rehabilitation of the severely deficient edentulous maxilla. Cochrane Database Syst Rev 2005; 19: CD004151.

\section{Appendix 1 \\ Questionnaire}

Do you carry out maxillary resections?

Yes $\quad \square \quad$ No

How many cases do you treat a year?

Between 1-5

Between 6-10

Over 10

Do you carry out surgical reconstruction of the maxillary defect?

Yes

No

What percentage of cases are surgically reconstructed?

$0-25 \%$

$25-50 \%$

$50-75 \%$

75-100\%

What type of flap do you use to reconstruct the surgical defect?

Radial forearm graft

Temporalis

DCIA

Scapula

Other (Please specify)

$\square$

a specify)

in your Trust?

No

ENT

Plastics

General surgery

Are all your oncology patients seen on a multidisciplinary team clinic?

Yes

No

Following surgical reconstruction, are the patients dentally rehabilitated?

Yes $\quad \square$

No

Do you have access to the services of a restorative dentist to assist with prosthetic rehabilitation? Yes

No - Y

If yes, what grade of dentist do you have in your team?

Consultant in restorative dentistry $\square$

Associate specialist

Staff grade

Senior house officer

Clinical assistant

Other

$\square$

$\square$

Do you use dental implants to reconstruct patients' dentition?

Yes

What design of dental implants do you routinely use for prosthetic reconstruction?

Nobel Biocare

Astra

$3 i$

Straumann

Other

$\square$

Are the implants placed by the surgeon or by the restorative dentist?

Maxillofacial surgeon

Restorative dentist

Depends

Not applicable

Are dental implants placed at the time of primary surgery?

Yes, always

Yes, sometimes

No

Do you use zygomatic implants for maxillary prosthetic reconstruction?

Yes

No

Do you have access to hyperbaric oxygen therapy (HBO) for patients with osteoradionecrosis or requiring dental implants?

Yes

No

Do you routinely use HBO for patients who have undergone radiotherapy who are scheduled for dental implants?

Yes

No 
26. Granström $G$, Jacobsson M, Tjellström A. Titanium implants in irradiated tissue: benefits from hyperbaric oxygen. Int J Oral Maxillofac Implants 1992; 7: 15-25.

27. Gerlach N L, Barkhuysen R, Kaanders J H, Janssens G 0, Sterk W, Merkx M A. The effect of hyperbaric oxygen therapy on quality of life in oral and oropharyngeal cancer patients treated with radiotherapy. Int J Oral Maxillofac Surg 2008; 37: 255-259.

28. Coulthard P, Esposito M, Worthington $H V$, Jokstad A. Therapeutic use of hyperbaric oxygen for irradiated dental implant patients: a systematic review. J Dent Educ 2003; 67: 64-68.

29. Kanatas A N, Lowe D, Harrison J, Rogers S N. Survey of the use of hyperbaric oxygen by maxillofacial oncologists in the UK. Br J Oral Maxillofac Surg 2005; 43: 219-225.

30. Marx R E, Johnson R P, Kline S N. Prevention of osteoradionecrosis: a randomized prospective clinical trial of hyperbaric oxygen versus penicillin. J Am Dent Assoc 1985; 111: 49-54.

31. Annane D, Depondt J, Aubert P et al. Hyperbaric oxygen therapy for radionecrosis of the jaw: a randomized, placebo-controlled, double-blind trial from the ORN96 study group. J Clin Oncol 2004; 22: 4893-4900.

32. Franzén L, Rosenquist J B, Rosenquist K I, Gustafsson I. Oral implant rehabilitation of patients with oral malignancies treated with radiotherapy and surgery without adjunctive hyperbaric oxygen. Int J Oral Maxillofac Implants 1995; 10: 183-187.

33. Department of Health. A policy framework for commissioning cancer services: a report by the Expert Advisory Group on Cancer to the Chief Medical Officers of England and Wales. London: Department of Health, 1995.
34. Brown J S, Magennis P, Rogers S N, Cawood J I, Howell R, Vaughan E D. Trends in head and neck microvascular reconstructive surgery in Liverpoo (1992-2001). Br J Oral Maxillofac Surg 2006; 44: 364-370.

35. Adell R, Svensson B, Bågenholm T. Dental rehabilitation in 101 primarily reconstructed jaws after segmental resections - possibilities and problems. An 18-year study. J Craniomaxillofac Surg 2008; 36: 395-402.

36. Assael $L A$. Dental implant education and practice in oral and maxillofacial surgery: 25 years of progress. J Oral Maxillofac Surg 2008; 66: 611-612.

37. Melo M D, McGann G, Obeid G. Survey of implant training in oral and maxillofacial surgery residency programs in the United States. J Oral Maxillofac Surg 2007; 65: 2554-2558.

38. Butterworth C J, Baxter A M, Shaw M J, Bradnock G. The provision of dental implants in the National Health Service hospital dental services - a national questionnaire. Br Dent J 2001; 190: 93-96.

39. Vartoukian S R, Algraffee H. Does the referral and selection for NHS-funded dental implant treatment in the UK follow national guidelines? Ann R Coll Surg Eng/ 2007; 89: 247-251.

40. Esposito M, Murray-Curtis L, Grusovin M G, Coulthard $\mathrm{P}$, Worthington $\mathrm{H} \mathrm{V}$. Interventions for replacing missing teeth: different types of denta implants. Cochrane Database Syst Rev 2007 17: CD003815.

41. Meirelles L, Currie F, Jacobsson M, Albrektsson $T$, Wennerberg $A$. The effect of chemical and nanotopographical modifications on the early stages of osseointegration. Int J Oral Maxillofac Implants 2008; 23: 641-647.

42. Office for National statistics. One and five year survival of patients diagnosed in 1991-95 and
1996-99: less common cancers, sex and age, England \& Wales. London: Office of National Statistics, 2005. http://www.statistics.gov.uk/ STATBASE/ssdataset.asp?vlnk $=8904$

43. Barclay C. The benefits of online implant recognition systems. Pract Proced Aesthet Dent 2008; 20: 500-502.

44. Sclaroff A, Haughey B, Gay W D, Paniello R. Immediate mandibular reconstruction and placement of dental implants. At the time of ablative surgery. Oral Surg Oral Med Oral Pathol 1994; 78: 711-717.

45. Nelson K, Heberer S, Glatzer C. Survival analysis and clinical evaluation of implant-retained prostheses in oral cancer resection patients over a mean follow-up period of 10 years. J Prosthet Dent 2007; 98: 405-410.

46. Schmidt B L, Pogrel M A, Young C W, Sharma A. Reconstruction of extensive maxillary defects using zygomaticus implants. J Oral Maxillofac Surg 2004; 62: 82-89.

47. Landes C A. Zygoma implant-supported midfacial prosthetic rehabilitation: a 4-year follow-up study including assessment of quality of life. Clin Oral Implants Res 2005; 16: 313-325.

48. Esposito M, Grusovin M G, Patel S, Worthington H V , Coulthard P. Interventions for replacing missing teeth: hyperbaric oxygen therapy for irradiated patients who require dental implants. Cochrane Database Syst Rev 2008; 23: CD003603.

49. Schoen P J, Raghoebar G M, Bouma J et al. Rehabilitation of oral function in head and neck cancer patients after radiotherapy with implant-retained dentures: effects of hyperbaric oxygen therapy. Oral Oncol2007; 43: 379-388. 